\title{
Intravitreal Aflibercept Versus Ranibizumab for Wet Age-Related Macular Degeneration: A Cost-Effectiveness Analysis
}

\author{
Luis Hernandez, MSc; Tereza Lanitis, MSc; Clifford Cele, BA; Hector Toro-Diaz, PhD; \\ Andrea Gibson, PhD; and Andreas Kuznik, PhD
}

\begin{abstract}
BACKGROUND: Age-related macular degeneration (AMD) is the leading cause of vision loss in the United States. The most severe vision loss occurs in patients with neovascular AMD, known as wet AMD (wAMD). The most commonly used antivascular endothelial growth factor (VEGF) therapies approved by the FDA to treat patients with wAMD are ranibizumab, $0.5 \mathrm{mg}$ administered by intravitreal injection once a month (approximately every 28 days), and intravitreal aflibercept injection (IAI), 2 mg every 4 weeks (monthly) for the first 12 weeks ( 3 months), followed by IAI 2 mg once every 8 weeks ( 2 months). Given the similar efficacy and safety profiles between IAI and ranibizumab, their associated costs and comparative cost-effectiveness are key factors in determining which one represents a more rational investment of scarce health care resources to help address the increasing cost of prescription drugs in the United States, a source of concern for patients, prescribers, payers, and policymakers.
\end{abstract}

OBJECTIVE: To assess the cost-effectiveness of intravitreal aflibercept injection $2 \mathrm{mg}$ every 8 weeks after 3 initial monthly doses (IAI 2q8) versus ranibizumab $0.5 \mathrm{mg}$ monthly (Rq4) and pro re nata (PRN) in the treatment of patients with wAMD from a U.S. payer perspective.

METHODS: A Markov cohort model was developed to estimate the lifetime quality-adjusted life-years (QALYs) and costs of treating patients with wAMD with IAI 2q8, Rq4, and ranibizumab PRN. The model considered changes in best-corrected visual acuity in the affected and fellow eyes over time, and the effect of blindness on mortality. Efficacy for IAI 2q8 and Rq4 was from VIEW 1 and VIEW 2 studies and from the Comparison of AMD Treatments Trials for ranibizumab PRN. Utilities and costs (in 2016 U.S. dollars) were from published literature. Health outcomes and costs were discounted at an annual rate of $3 \%$.

RESULTS: Over a lifetime, IAI 2 q8 provided equal health benefits with Rq4 (5.44 QALYs) at a lower total cost $(\$ 33,745$ vs. $\$ 48,031)$ as a result of fewer injections. IAI 2 q8 yielded slightly greater $Q A L Y$ s versus ranibizumab PRN (5.44 vs. 5.40$)$ at a slightly higher cost $(\$ 33,745$ vs. $\$ 33,652)$, with an incremental cost per QALY gained of $\$ 2,583$. Results were sensitive to variations in drug acquisition costs and number of injections of both drugs and the baseline age of the cohort.

CONCLUSIONS: IAI 2q8 can be cost saving and cost-effective compared with Rq4 and ranibizumab PRN for the treatment of WAMD in the United States.

J Manag Care Spec Pharm. 2018;24(7):608-16

Copyright @ 2018, Academy of Managed Care Pharmacy. All rights reserved.

\section{What is already known about this subject}

Age-related macular degeneration (AMD) is the leading cause of vision loss in the United States, with the most severe vision loss occurring in patients with neovascular AMD, known as wAMD Antivascular endothelial growth factor (VEGF) therapy has changed the paradigm of wAMD treatment.

Intravitreal aflibercept injection (IAI) and ranibizumab are the most commonly used therapies approved by the FDA for the treatment of wAMD.

\section{What this study adds}

This study compares the cost-effectiveness of IAI 2 mg once every 8 weeks after 3 initial monthly doses (2q8) versus ranibizumab in the treatment of wAMD from a U.S. payer perspective.

Given the similar efficacy and safety profiles of IAI and ranibizumab, their associated costs and comparative cost-effectiveness are key factors in determining which one represents a more rational investment of scarce health care resources.

IAI (2q8) reduces the overall treatment costs compared with ranibizumab in the treatment of wAMD in the United States, and outcomes suggest potential improvements in patients' quality of life.

A ge-related macular degeneration (AMD) is the leading cause of vision loss in the United States, ${ }^{1,2}$ affecting more than 1.75 million people. ${ }^{2,3}$ AMD is most common among elderly white Americans, affecting more than 19\% of white Americans who are $\geq 65$ years. ${ }^{3}$ Because of the aged population, it is estimated that by 2020, nearly 3 million people in the United States will experience visual impairment due to AMD. ${ }^{2}$

There are several types of AMD. ${ }^{4}$ The most severe vision loss occurs in patients with neovascular AMD, known as wAMD. ${ }^{5}$ wAMD involves choroidal neovascularization (CNV) and associated retinal edema; blood vessels under the macula start to leak or bleed, affecting central vision and, in turn, activities of daily living, such as reading, recognizing faces, watching television, and driving. ${ }^{5}$

Antivascular endothelial growth factor (VEGF) therapy has changed the paradigm of wAMD treatment. ${ }^{6,7}$ VEGF has been suggested to be the main driver of $\mathrm{CNV}$ and associated edema observed in patients with wAMD, as it is an important modulator of angiogenesis. ${ }^{8}$ Anti-VEGF treatment via intravitreal 
injection limits the leaking or bleeding of submacular vessels, with the potential of restoring a patient's vision. ${ }^{7}$ The most commonly used anti-VEGF therapies approved by the U.S. Food and Drug Administration (FDA) for the treatment of patients with wAMD are ranibizumab $0.5 \mathrm{mg}$ administered by intravitreal injection once a month (approximately every 28 days), and intravitreal aflibercept injection (IAI) 2 mg every 4 weeks (monthly) for the first 12 weeks ( 3 months), followed by IAI $2 \mathrm{mg}$ once every 8 weeks ( 2 months). ${ }^{9,10}$ Ranibizumab and IAI were approved by the FDA and introduced in the U.S. market in 2006 and 2011, respectively. ${ }^{7}$

The VEGF Trap-Eye: Investigation of efficacy and safety in wAMD studies (VIEW 1 and VIEW 2)-phase III prospective, double-masked, multinational, parallel-group, active controlled randomized clinical trials-compared IAI $0.5 \mathrm{mg}$ monthly, 2 mg monthly, and 2 mg every 2 months after 3 initial monthly doses with ranibizumab $0.5 \mathrm{mg}$ monthly. In both trials, all 3 IAI regimens were statistically noninferior to monthly ranibizumab in preventing moderate visual acuity loss at 1 year and had a similar safety profile. ${ }^{5}$

Patients receiving ranibizumab $0.5 \mathrm{mg}$ may be treated pro re nata (PRN; i.e., as needed) with 3 monthly doses followed by less frequent dosing with regular assessment. ${ }^{10}$ The Comparison of AMD Treatments Trials (CATT) study showed that ranibizumab $0.5 \mathrm{mg}$ PRN with monthly evaluation had effects on vision that were equal to those of ranibizumab $0.5 \mathrm{mg}$ administered monthly. ${ }^{11}$

Given the similar efficacy and safety profiles between IAI and ranibizumab, their associated costs and comparative cost-effectiveness are key factors in determining which one represents a more rational investment of scarce health care resources. The increasing cost of prescription drugs in the United States is a source of concern for patients, prescribers, payers, and policymakers. ${ }^{12}$ Some strategies to address high prices include generating more evidence about comparative cost-effectiveness of therapeutic alternatives, and educating patients, prescribers, payers, and policymakers more effectively about these choices. ${ }^{12}$

We therefore assessed the long-term clinical efficacy and economic consequences and cost-effectiveness of IAI $2 \mathrm{mg}$ every 4 weeks (monthly) for the first 12 weeks ( 3 months), followed by IAI 2 mg once every 8 weeks (IAI 2q8) compared with ranibizumab $0.5 \mathrm{mg}$ monthly (Rq4) and ranibizumab $0.5 \mathrm{mg}$ PRN, in the treatment of patients with wAMD from a U.S. payer perspective.

\section{Methods}

\section{Model Structure}

An economic model using a Markov cohort approach was developed in Microsoft Excel 2016 (Microsoft, Redmond, WA) to model the lifetime transitions of a cohort of patients with wAMD through mutually exclusive health states based on involvement of wAMD (single eye vs. both eyes involvement), patients' treatment status (on- and off-treatment), and bestcorrected visual acuity (BCVA). The model includes mutually exclusive health states for patients with single eye involvement versus involvement in both eyes, and for patients receiving anti-VEGF treatment in 1 or 2 eyes versus patients receiving no active treatment (Figure 1A). Similar to previously published economic models and reimbursement submissions to health technology assessment agencies, ${ }^{13-15} 5$ substates were used within each eye to define BCVA, measured using the Early Treatment Diabetic Retinopathy Study letters score (Figure 1B), the gold standard test for measuring and monitoring visual acuity in patients with AMD. ${ }^{16-20}$

All patients entering the model were assumed to require treatment for at least 1 eye affected with wAMD (treated eye). A proportion of the cohort was assumed to have WAMD in the second eye (fellow eye), thus initiating bilateral treatment. The remainder of patients, whose fellow eye was unaffected by wAMD, initiated unilateral treatment. However, patients who initiated unilateral treatment were at risk of developing wAMD in the fellow eye and could receive treatment in the fellow eye at any point during the model time horizon. In these patients, treatment was initiated at the time of fellow eye involvement and therefore at a later time as compared with treatment initiation in the first affected eye. Involvement of WAMD, treatment status, and BCVA were updated every 6 months in a recurring fixed interval (model cycle).

During each model cycle, treated eyes could (a) remain at the current level of BCVA, (b) improve by an average of 15 letters, or (c) worsen by an average of 15 letters. Eyes at the lowest BCVA level ( $<35$ letters) could only remain in this health state or improve to the next BCVA level (36-50 letters). Eyes at the highest BCVA level (> 79 letters) could only remain in that health state or worsen to the previous BCVA level (65-79 letters). Treatment in each eye was assumed to be provided for a maximum of 2 years (approximate duration of VIEW studies) and could be discontinued earlier. ${ }^{5,21}$

BCVA in untreated eyes affected with wAMD (i.e., eyes discontinuing treatment) could remain stable or decline following the natural history of the disease. BCVA in eyes unaffected by WAMD was assumed to remain stable. No improvements in BCVA were allowed during the off-treatment phase.

Each eye was modeled independently of the other, using the same levels of BCVA, as shown in Figure 1B. Affected fellow eyes were assumed to receive the same treatment as the treated eye as soon as the disease developed (i.e., in the same cycle when involvement happened) and experienced the same benefits from treatment.

In addition to changes in wAMD involvement, treatment, and BCVA, background mortality was also modeled, considering the age of the cohort, increased risk in mortality due to WAMD and its associated comorbidities, and blindness $\left(\mathrm{BCVA}<35\right.$ in the better-seeing eye [BSE]). ${ }^{22,23}$ 


\section{FIGURE 1 Model Structure}

\section{A. Health States}

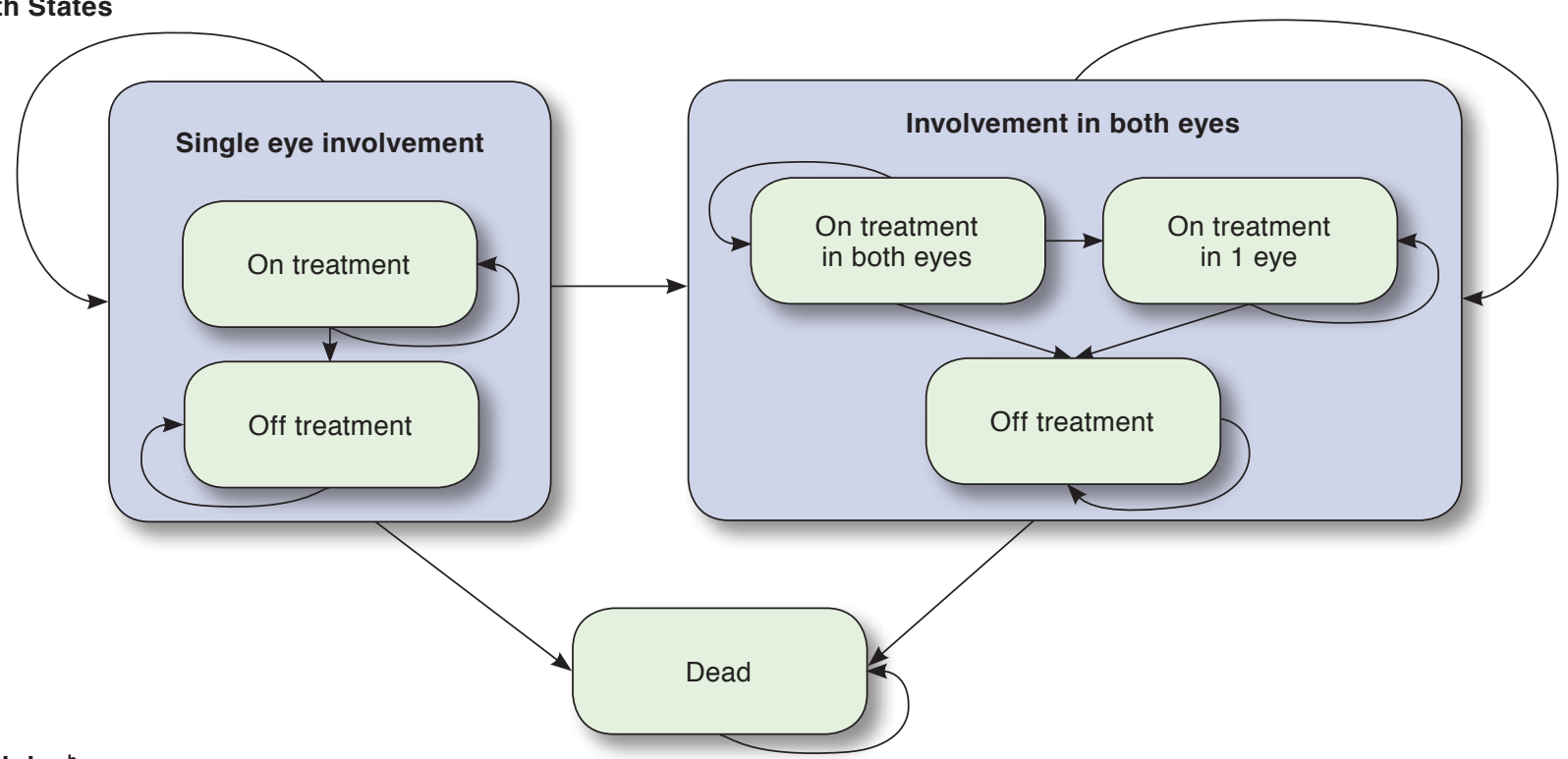

B. Substates ${ }^{b}$

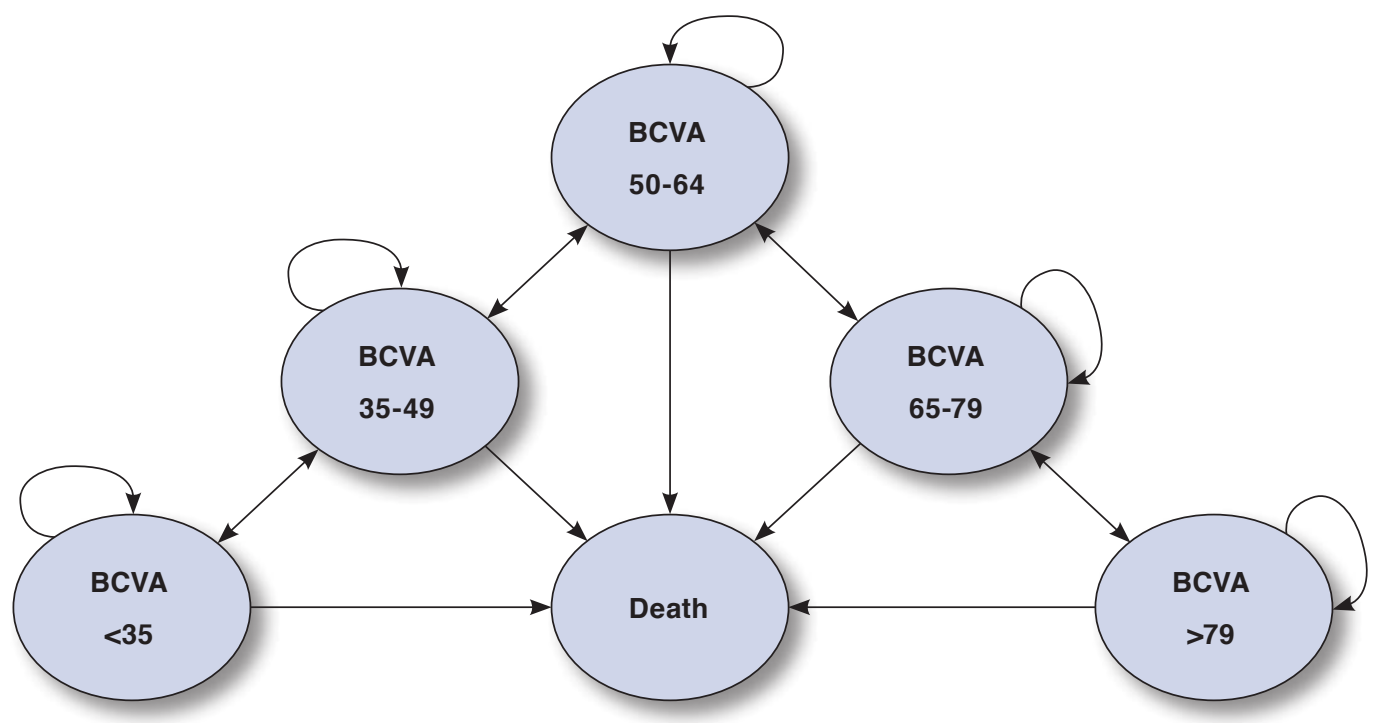

${ }^{a}$ Ovals represent health states. Solid line arrows indicate allowed transitions after every cycle.

bSubstates are modeled within each health state in Figure $1 \mathrm{~A}$, except for death.

$B C V A=$ best-corrected visual acuity (measured using the Early Treatment Diabetic Retinopathy Study letters score).

As patients moved between states, they accrued state- and event-specific costs (e.g., blindness cost when $B C V A<35$ ) and were assigned utilities to estimate quality-adjusted life-years (QALYs). Utilities were assigned based on the BCVA of the BSE. The distribution of BCVA in the BSE took into account the current state of both eyes.

\section{Inputs and Data Sources}

Table 1 details distributions of BCVA in each eye, as well as utility values associated with each BCVA state (based on the BSE). Inputs relating to efficacy and costs associated with treatment are shown in Table 2.

Patients entered the model with a mean age of 76 years, with the BCVA distribution in each eye based on those observed in 


\begin{tabular}{|c|c|c|c|}
\hline \multirow[b]{2}{*}{ BCVA Level } & \multicolumn{2}{|c|}{$\begin{array}{l}\text { Initial Distribution (\%) } \\
\text { PSA: Dirichlet Distribution }\end{array}$} & \multirow{2}{*}{$\begin{array}{c}\text { Utility Score for } \\
\text { BSE }^{44}(95 \% \mathrm{CI}) \\
\text { PSA: Beta Distribution }\end{array}$} \\
\hline & Treated Eye 5 & Fellow Eye ${ }^{48}$ & \\
\hline$<35$ & 11.78 & 14.79 & $0.610 \quad(0.57-0.65)$ \\
\hline $35-49$ & 19.13 & 4.50 & $0.665 \quad(0.62-0.71)$ \\
\hline $50-64$ & 45.12 & 10.04 & $0.720 \quad(0.67-0.77)$ \\
\hline $65-79$ & 23.97 & 33.14 & $0.810 \quad(0.78-0.84)$ \\
\hline$>79$ & 0.00 & 37.53 & $0.880 \quad(0.85-0.91)$ \\
\hline
\end{tabular}

$B C V A=$ best-corrected visual acuity; $B S E=$ better-seeing eye; $C I=$ confidence interval; $P S A=$ probabilistic sensitivity analysis.

patients in the VIEW studies. ${ }^{5}$ In $19 \%$ of patients, wAMD was assumed to be present in both eyes at baseline, ${ }^{5}$ while in the remainder, fellow-eye involvement could occur throughout the model time horizon. The annual risk of fellow-eye involvement $(8.10 \%, 95 \%$ confidence interval $[\mathrm{CI}]=4.9 \%-11.9 \%)$ was obtained from a published systematic review and meta-analysis and assumed to be independent of treatment. ${ }^{24}$

Transitions between health states for each treatment were based on the percentage of patients gaining or losing $\geq 15$ letters. For patients receiving IAI $2 \mathrm{q} 8$, the percentages of patients gaining or losing $\geq 15$ letters were obtained from the VIEW studies. ${ }^{5}$ For patients receiving Rq4 or ranibizumab PRN, relative effects were applied to the percent gain/loss observed in the IAI $2 \mathrm{q} 8 \mathrm{arm}$. Relative efficacy for Rq 4 versus IAI $2 \mathrm{q} 8$ was obtained from the VIEW studies. ${ }^{5}$ As observed in the VIEW studies, a slightly numerically higher number of patients on $\mathrm{Rq} 4$ experienced improvement and worsening $\geq 15$ letters compared with IAI 2q8. Relative efficacy for ranibizumab PRN was derived from the CATT study via Bucher indirect comparison versus IAI $2 \mathrm{q} 8$, using $\mathrm{Rq} 4$ as common comparator. ${ }^{11,25}$ On the basis of the BCVA results from the second yearof theVIEWstudiesand theopen-labelextension of theVIEW 1 study (2 additional years of follow-up), ${ }^{21,26}$ it was assumed that patients remained at the current level of BCVA after the first year of treatment, unless treatment discontinuation occurred (Table 2). After treatment discontinuation, BCVA did not improve, rather it remained stable or worsened. The natural history annual risk of worsening $\geq 15$ letters (8.38\%, $95 \% \mathrm{CI}=4.83 \%-12.26 \%$ ) was obtained from a published systematic review and meta-analysis and was assumed to be independent of treatment. ${ }^{24}$

The annual risk of treatment discontinuation for IAI 2q8 was obtained from the VIEW studies. ${ }^{5,21}$ The risk of treatment discontinuation for both ranibizumab regimens was assumed to be the same as that of IAI $2 \mathrm{q} 8$, as they were not reported in the CATT study and there was no statistically significant difference between IAI 2q8 and Rq4 in the VIEW studies.
Background mortality was modeled based on age-specific U.S. life tables. ${ }^{27}$ A hazard ratio of 1.76 (95\% CI $=1.20-12.57$ ) was applied to background risk of death to reflect increased mortality associated with wAMD. ${ }^{22}$ In patients with bilateral blindness, this hazard ratio was multiplied by 1.23 (95\% CI $=1.16-1.31)$ to reflect further excess mortality due to blindness. ${ }^{23}$

Costs (in 2016 U.S. dollars) of drug acquisition and monitoring (Table 2) and the annual cost associated with blindness $(\$ 7,076.79$, range $=\$ 5,661.43-\$ 8,492.15)$ were obtained from public databases and literature. ${ }^{28}$ Drug acquisition costs were based on wholesale acquisition cost (WAC; from RED BOOK Online. 2016. Truven Health Analytics. Accessed July 20, 2016). Using the WAC reflects the manufacturer's list price of the drug when sold to the wholesalers, and it is a better representation of actual market prices than other benchmarks, such as the average wholesale price, which is usually an elevated drug price that is rarely what is actually paid. ${ }^{29,30}$

\section{Analyses}

The model followed patients throughout their lifetimes. Health outcomes (e.g., life-years and QALYs) and cost outcomes (e.g., drug acquisition and monitoring costs and cost of blindness) were discounted at an annual rate of $3.0 \% .^{31}$ The relative benefits of IAI 2 q8 versus both ranibizumab regimens were assessed using the incremental cost-effectiveness ratio (ICER), representing the additional costs associated with IAI $2 \mathrm{q} 8$ to achieve an additional QALY compared with Rq4 and ranibizumab PRN. A treatment was deemed cost-effective at ICERs below a willingness-to-pay (WTP) threshold of $\$ 50,000$ per QALY gained. ${ }^{32}$

The predictive validity of the model was examined by comparing the trend of the mean BCVA over time predicted by the model with the trend of the mean BCVA reported in the CATT extension study over 5 years. ${ }^{33}$ Trends predicted from the model were similar to those observed in the CATT extension study, with mean BCVA increasing in the first year and declining thereafter, reaching baseline values at approximately 5 years. ${ }^{33}$

Deterministic sensitivity analyses (DSAs) were conducted to evaluate how the model results varied in relation to changes in all model parameters. Where possible, 95\% CIs were used to vary parameters. Where these were not available, parameters were varied by $\pm 20 \%$.

In order to account for statistical uncertainties of multiple key parameters, probabilistic sensitivity analyses (PSAs) were performed by simultaneously varying parameters using probability distributions. Parameters varied included the baseline BCVA level distribution for the treated and fellow eyes, the percentage of patients with fellow-eye involvement, the annual risk of fellow-eye involvement, both the risk of improvement and worsening for each treatment, the natural history annual risk of 
TABLE 2 Treatment-Related Model Inputs: Efficacy and Costs

\begin{tabular}{|c|c|c|c|c|c|c|}
\hline \multirow{2}{*}{$\begin{array}{l}\text { Parameter } \\
\text { PSA Distribution }\end{array}$} & \multicolumn{2}{|c|}{ IAI 2q8 Value (Range) } & \multicolumn{2}{|c|}{ Rq4 Value (Range) } & \multicolumn{2}{|c|}{$\begin{array}{c}\text { Ranibizumab } 0.5 \text { mg PRN Value } \\
\text { (Range) }\end{array}$} \\
\hline & Year 1 & Year 2+ & Year 1 & Year $2+$ & Year 1 & Year $2+$ \\
\hline Prob. improving $\geq 15$ letters (normal) & $\begin{array}{c}0.3514 \\
(0.3115-0.3923)^{5}\end{array}$ & $0.0^{\mathrm{a}}$ & & & & \\
\hline Prob. worsening $\geq 15$ letters (normal) & $\begin{array}{c}0.0467 \\
(0.0305-0.0661)^{5}\end{array}$ & $0.0^{\mathrm{a}}$ & & & & \\
\hline RR improving $\geq 15$ letters $^{b}$ (normal) & & & $\begin{array}{c}1.021 \\
(0.869-1.199)^{5}\end{array}$ & $1.0^{\mathrm{c}}$ & $\begin{array}{c}0.714 \\
(0.527-0.969) \mathrm{d}\end{array}$ & $1.0^{\mathrm{c}}$ \\
\hline RR worsening $\geq 15$ letters $^{\mathrm{b}}$ (normal) & & & $\begin{array}{c}1.193 \\
(0.712-2.001)^{5}\end{array}$ & $1.0^{\mathrm{c}}$ & $\begin{array}{c}0.678 \\
(0.281-1.637)^{\mathrm{d}} \\
\end{array}$ & $1.0^{\mathrm{c}}$ \\
\hline Annual discontinuation risk (normal) & $\begin{array}{c}0.0863 \\
(0.0755-0.0977)^{5}\end{array}$ & $\begin{array}{c}0.0903 \\
(0.0788-0.1025)^{21}\end{array}$ & $\begin{array}{c}0.0863 \\
(0.0755-0.0977)^{\mathrm{c}} \\
\end{array}$ & $\begin{array}{c}0.0903 \\
(0.0788-0.1025)^{\mathrm{c}} \\
\end{array}$ & $\begin{array}{c}0.0863 \\
(0.0755-0.0977)^{\mathrm{c}} \\
\end{array}$ & $\begin{array}{c}0.0903 \\
(0.0788-0.1025)^{c}\end{array}$ \\
\hline Number of injections & $\begin{array}{c}7.5 \\
(6-8)^{5}\end{array}$ & $\begin{array}{c}4.5 \\
(3.6-5.4)^{33}\end{array}$ & $\begin{array}{c}11.7 \\
(11.5-11.9)^{11}\end{array}$ & $\begin{array}{c}4.5 \\
(3.6-5.4)^{c}\end{array}$ & $\begin{array}{c}6.9 \\
(5.5-8.3)^{11}\end{array}$ & $\begin{array}{c}4.5 \\
(3.6-5.4)^{c}\end{array}$ \\
\hline Monitoring costs (annual), \$e & $\begin{array}{c}1,509 \\
(1,207-1,811)\end{array}$ & $\begin{array}{c}738 \\
(591-886) \\
\end{array}$ & $\begin{array}{c}2,198 \\
(1,758-2,638)\end{array}$ & $\begin{array}{c}738 \\
(591-886)^{c}\end{array}$ & $\begin{array}{c}1,410 \\
(1,128-1,693)\end{array}$ & $\begin{array}{c}738 \\
(591-886)^{c}\end{array}$ \\
\hline Acquisition costs (per injection), $\$^{f}$ & \multicolumn{2}{|c|}{$\begin{array}{c}1,850 \\
(1,480-2,220) \\
\end{array}$} & \multicolumn{2}{|c|}{$\begin{array}{c}1,950 \\
(1,560-2,340) \\
\end{array}$} & \multicolumn{2}{|c|}{$\begin{array}{c}1,950 \\
(1,560-2,340) \\
\end{array}$} \\
\hline \multicolumn{7}{|c|}{ 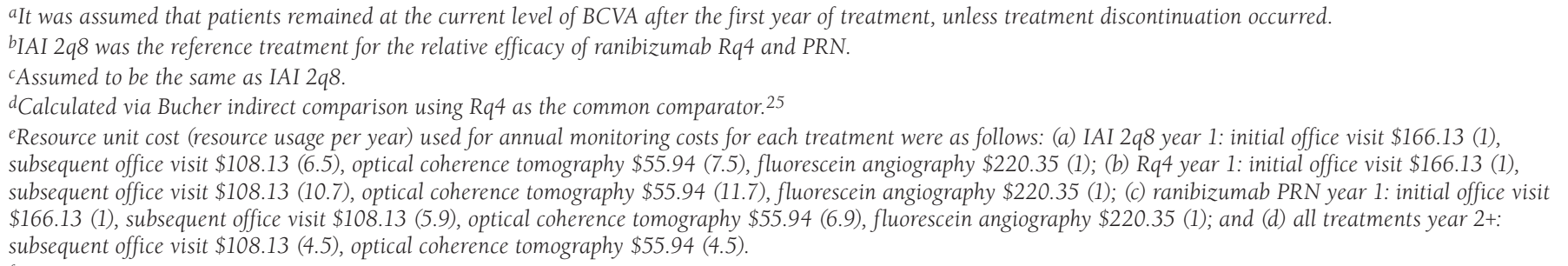 } \\
\hline
\end{tabular}

worsening, the annual risk of discontinuation, the utilities by BCVA level, and the mortality hazard ratios due to wAMD and blindness. Drug acquisition costs were not varied, as they are not subject to parameter uncertainty. The PSA was carried out running 1,000 simulations.

\section{Results}

As shown in Table 3, over a lifetime, IAI 2q8 provided equal health benefits with Rq4 (5.44 QALYs) at a lower total cost $(\$ 33,745$ vs. $\$ 48,031)$. This cost savings occurred because patients on IAI 2 q8 received fewer injections over the 2 years of treatment versus patients on $\mathrm{Rq} 4$ (Table 2), and because the cost per injection and the monitoring costs during the first year were lower for patients on IAI 2 q8. IAI 2 q8 yielded greater QALYs compared with ranibizumab PRN (5.440 vs. 5.404) at a higher cost $(\$ 33,745$ vs. $\$ 33,652)$, with an incremental cost per QALY gained of $\$ 2,583$ (i.e., IAI 2q8 was cost-effective vs. ranibizumab PRN). The greater QALYs for patients on IAI 2q8 were a result of patients experiencing a higher likelihood of improvement, as shown in Table 2.
The DSA showed that IAI 2 q8 was cost-saving or dominant (i.e., less expensive and more effective) versus $\mathrm{Rq} 4$ in most scenarios, except when the year 1 relative risk (RR) of improvement for $\mathrm{Rq} 4$ was increased to the upper bound of its $95 \%$ CI (i.e., $\mathrm{Rq} 4$ performed better than in the base case), and the year $1 \mathrm{RR}$ of worsening for $\mathrm{Rq} 4$ was decreased to the lower bound of its 95\% CI (i.e., Rq4 performed better than in the base case). In these scenarios, IAI 2q8 was less expensive and less effective.

The DSA showed that IAI 2q8 was cost-effective or dominant versus ranibizumab PRN in most scenarios, except in the following scenarios in which IAI 2q8 was more expensive and effective but not cost-effective:

- The drug acquisition costs for IAI 2q8 was increased by $20 \%$ (ICER $=\$ 167,000)$ or decreased by $20 \%$ for ranibizumab PRN (ICER $=\$ 166,583)$.

- The year $1 \mathrm{RR}$ of improvement for ranibizumab PRN was increased to the upper bound of its 95\% CI (ICER $=\$ 76,000)$.

- The number of injections during year 1 for IAI 2q8 was increased by $20 \%(\mathrm{ICER}=\$ 112,639)$ or decreased by $20 \%$ for ranibizumab $0.5 \mathrm{mg}$ PRN (ICER=\$109,306). 


\begin{tabular}{|c|c|c|c|}
\hline $\begin{array}{l}\text { Outcome (Discounted } \\
\text { per Patient, Unless } \\
\text { Otherwise Specified) }\end{array}$ & IAI $2 q 8$ & $\mathrm{Rq} 4$ & $\begin{array}{c}\text { Ranibizumab } \\
0.5 \mathrm{mg} \text { PRN }\end{array}$ \\
\hline \multicolumn{4}{|l|}{ Clinical outcomes } \\
\hline Life-years (undiscounted) & 8.076 & 8.075 & 8.071 \\
\hline QALYs & 5.440 & 5.440 & 5.404 \\
\hline \multicolumn{4}{|l|}{ Cost outcomes, \$ } \\
\hline Treatment acquisition & 29,593 & 42,886 & 29,519 \\
\hline Treatment monitoring & 3,022 & 4,006 & 2,881 \\
\hline Blindness & 1,130 & 1,139 & 1,251 \\
\hline Total cost, $\$$ & 33,745 & 48,031 & 33,652 \\
\hline \multicolumn{4}{|c|}{ Incremental outcomes (IAI 2 q8 vs. comparator) } \\
\hline QALYS & & 0.0 & 0.036 \\
\hline Costs, $\$$ & & $-14,286$ & 93 \\
\hline $\begin{array}{l}\text { ICER (\$ per QALY } \\
\text { gained) }\end{array}$ & & $\begin{array}{l}\text { Less costly, } \\
\text { equal efficacy }\end{array}$ & 2,583 \\
\hline \multicolumn{4}{|c|}{$\begin{array}{l}\text { ETDRS = Early Treatment Diabetic Retinopathy Study; IAI } 2 q 8=\text { intravitreal } \\
\text { aflibercept injection } 2 \mathrm{mg} \text { every } 4 \text { weeks (monthly) for the first } 12 \text { weeks } \\
(3 \text { months), followed by IAI } 2 \mathrm{mg} \text { once every } 8 \text { weeks; ICER=incremental cost- } \\
\text { effectiveness ratio; PRN = pro re nata (i.e., as needed); QALYs =quality-adjusted } \\
\text { life-years; Rq4 = ranibizumab } 0.5 \mathrm{mg} \text { monthly. }\end{array}$} \\
\hline
\end{tabular}

- The number of injections during subsequent years of treatment for IAI 2 q8 was increased by $20 \%$ (ICER $=\$ 56,944$ ) or decreased by $20 \%$ for ranibizumab $0.5 \mathrm{mg}$ PRN (ICER $=\$ 59,681)$.

The PSA results indicate that compared with Rq4, IAI 2 q8 was cost-effective in all simulations, being dominant in $46 \%$ of them. Compared with ranibizumab PRN, IAI 2q8 was costeffective in $98 \%$ of all simulations, less expensive and less effective in approximately $2 \%$, and dominated in $<1 \%$. The PSA indicated that IAI $2 \mathrm{q} 8$ would be the preferred treatment choice, representing the maximum net benefit over both ranibizumab regimens across all WTP thresholds (range \$25,000-\$625,000 per QALY gained).

\section{Discussion}

To our knowledge, this is the first analysis to compare the costeffectiveness of IAI 2q8 versus ranibizumab in the treatment of wAMD from a U.S. payer perspective. These findings are consistent with earlier analyses conducted in other settings,which concluded that IAI 2q8 provided higher or similar QALYs gained to ranibizumab, producing lower or economically justifiable additional costs depending on the regimen examined. . $^{34-39}$

One study reported a marginally lower QALY gain for IAI $2 q 8$ versus Rq4 and ranibizumab PRN in the treatment of wAMD (2.15 vs. 2.15 and 2.16, respectively); however, their analysis was conducted over 5 years compared with a lifetime horizon in this analysis. ${ }^{40}$ Nonetheless, the study reported that IAI 2 q 8 provided cost savings versus both ranibizumab regimens ( $€ 36,030$ for IAI 2 q8 vs. $€ 74,837$ for Rq4 and $€ 45,491$ for PRN). Another study reported lower QALYs and higher costs for IAI versus ranibizumab, both administered PRN in the treatment of wAMD (5.73 and 5.77 total QALYs, and $£ 39,745$ and $£ 31,361$ for IAI vs. ranibizumab, respectively). ${ }^{41}$ This study was conducted from a United Kingdom perspective, and the cost per administration associated with ranibizumab was lower than that of IAI, in contrast to estimates used in this analysis based on U.S. list prices. In addition to the different regimens used in this study, the study assumed an almost $70 \%$ higher discontinuation rate associated with IAI versus ranibizumab, which resulted in a shorter duration of treatment and subsequent lower exposure to the benefits of anti-VEGF treatment in IAI-treated patients. In this analysis, we assumed that the risk of discontinuation was independent of treatment. Results from 96 weeks of the VIEW studies suggested that $14.8 \%$ of patients in the Rq4 arm had discontinued treatment, compared with $16.7 \%$ in the IAI 2 q8 arm. ${ }^{21}$ When implemented in this model, IAI 2 q8 still provided equal health benefits versus $\mathrm{Rq} 4$ at a lower cost $(\$ 33,691$ vs. $\$ 48,100)$.

This model attempts to overcome limitations associated with earlier analyses by explicitly considering involvement of wAMD in the fellow eye and its treatment. The majority of previous models have only considered a single eye, assuming in most cases that the BSE was the treated eye. ${ }^{15,42}$ Approximately a quarter of patients in the VIEW studies had their BSE treated, with the majority receiving treatment in the worst-seeing eye (WSE) ${ }^{38}$ Quality of life has been shown to be related to BCVA in the BSE, with limited evidence to support BCVA in the WSE as a determinant. ${ }^{43-47}$ Thus, modeling both eyes to determine the evolution of the BSE over time allows assigning utilities more accurately.

\section{Limitations}

Explicit modeling of the fellow eye required several assumptions, which may have limited our analysis. First, patient-level data were not available to the researchers. Therefore, in absence of information to assume otherwise, we assumed that BCVA in the fellow eye was independent of BCVA in the treated eye. One hypothesis is that if 1 eye has already suffered from wAMD, the second eye will be monitored frequently; thus, changes in BCVA will be noticeable early. Post hoc analysis of the VIEW studies, however, did not support this hypothesis. ${ }^{48}$ The mean BCVA in the treated eye was 53.7 when the baseline fellow-eye BCVA was above $\geq 50$ letters and 54 when the baseline felloweye BCVA was below $<50$ letters ${ }^{48}$ indicating that a positive or negative correlation in BCVA between the 2 eyes may not necessarily hold. Nonetheless, if either positive or negative correlation holds, the implication in the model would be classifying a larger proportion of patients in the bilateral blindness state, resulting in higher life-year and QALY gains for aflibercept. Thus the assumption used was considered conservative.

Second, because of paucity of data to inform the evolution of BCVA in the fellow eye, we assumed that when the fellow 
eye was affected with wAMD, BCVA would be similar to that of the treated eye at baseline and that treatment would commence immediately. This assumption is a simplification resulting from the cohort approach adopted. It is likely that BCVA in fellow eyes newly affected with wAMD would be less severe than that observed in the treated eyes at trial baseline, and that treatment would commence at a later time.

Had we modeled natural progression in the fellow eye as newly affected with wAMD and commencement of treatment at a later time, fellow-eye involvement would have a lesser effect on relative results between treatments, as benefits would occur later in time and be subject to higher discounting. To test the influence of this assumption, we examined results using a unilateral model, assuming fellow-eye BCVA is stable throughout the model time horizon to minimize the effects of the fellow eye. In this scenario, IAI 2q8 remained dominant versus Rq4, and the ICER versus ranibizumab PRN increased marginally to $\$ 4,075$ per QALY gained.

Further limitations may arise from simplifying assumptions adopted. We assumed that the risk of improving/worsening is the same for all eyes at any given cycle, regardless of the current BCVA level. Results from clinical studies consistently reported the overall change in patients' BCVA in terms of probability of improving/worsening the BCVA by 15 letters, but these results were not stratified based on the observed BCVA of the patients. Although this may affect absolute differences between treatments, the direction of incremental results should remain unaffected.

Further uncertainty stems from the efficacy assumptions used in this study (i.e., the probability of improving and worsening $\geq 15$ letters) informed from clinical trial results observed over a period of 1 year. As this study considered a lifetime horizon of analysis, the projections of the treatment efficacy after 1 year can be subject to great uncertainty. We assumed that patients remained at the current level of BCVA after the first year of treatment, unless treatment discontinuation occurred. After treatment discontinuation, BCVA did not improve, rather it remained stable or worsened based on the natural history annual risk of worsening $\geq 15$ letters. ${ }^{24}$ Our approach is consistent with other modeling studies in this therapeutic area, ${ }^{41,49}$ the results from the second year of the VIEW studies, and the open-label extension of the VIEW 1 study (2 additional years of follow-up). ${ }^{21,26}$

As presented in the Analyses section, the mean BCVA trends predicted from the model were similar to those observed in the CATT extension study, ${ }^{33}$ with mean BCVA increasing in the first year and declining thereafter, reaching baseline values at approximately 5 years. These findings are also consistent with those observed in the SEVEN-UP multicenter cohort study, assessing the long-term outcomes 7 to 8 years after initiation of intensive ranibizumab therapy. ${ }^{50}$

Our analysis used head-to-head data from the VIEW studies to compare IAI $2 q 8$ with Rq4. However, as no head-to-head comparisons exist between IAI 2q8 and ranibizumab PRN, we obtained relative effects for this comparison with a Bucher indirect comparison, ${ }^{25}$ using $\mathrm{Rq} 4$ as the common arm between the VIEW and CATT trials. Use of relative effects obtained from a meta-analysis including the CATT, HARBOR, and DETAIL trials of ranibizumab PRN did not alter the conclusions of our analysis, which resulted in a marginally higher ICER of \$5,064 per QALY gained for IAI 2q8 versus ranibizumab PRN. ${ }^{38}$

Further limitations may arise from paucity of data to inform treatment patterns. In the base case analysis (Table 3), we assumed that patients would be treated for a maximum of 2 years, consistent with the duration of observed data from the VIEW studies and the maximum duration assumed by the majority of previous evaluations. ${ }^{34-37,40}$ The optimal duration of treatment has not been established, and there is limited longterm evidence to inform the duration of treatment in patients with WAMD. As WAMD is a chronic disease, we examined longer treatment durations, up to lifetime.

In addition, visual gains achieved with IAI during 2 years of VIEW 1 were largely maintained by continued treatment with IAI $2 \mathrm{mg}$ over a period of 2 years in the open-label extension study. ${ }^{26}$ Increasing the treatment duration beyond 2 years resulted in larger cost savings versus both ranibizumab regimens, largely due to the lower cost per injection associated with IAI 2 q8 ( $\$ 1,850$ vs. $\$ 1,950)$ and anticipated a similar number of injections beyond 2 years. ${ }^{21,51}$ In addition, varying discontinuation rates between treatments did not alter the conclusions of our analyses.

The effect of retreatment and subsequent treatments following lack of improvement after 2 years of treatment or treatment discontinuation was not considered in the analyses. To appropriately model retreatment and subsequent treatments, treatment efficacy and discontinuation data are required for patients previously treated with specific anti-VEGF sequences. To our knowledge, such data are not available. In addition, although including this feature would better reflect clinical practice in real life, it could, however, mask the true value of a specific anti-VEGF evaluated in this analysis, as any benefits could result from other subsequent treatments. Accordingly, it was decided to exclude retreatment or subsequent lines of treatment in the current analysis. However, future analyses should examine the cost-effectiveness of different treatment sequences, including retreatment, to better reflect the treatment patterns of wAMD in actual practice.

Moreover, although anti-VEGF treatment is associated with adverse events, these were not modeled explicitly, as clinical evidence suggests that the safety profile for both comparators is similar. ${ }^{5,21,52}$ Earlier analyses that considered adverse events found that these had a negligible effect on cost-effectiveness results. ${ }^{15}$

Finally, this analysis focused on comparing the 2 most commonly used FDA-approved anti-VEGF agents. Bevacizumab is another anti-VEGF approved for several indications in 
oncology. ${ }^{53}$ Although bevacizumab is not indicated for wAMD, nor has it been approved by the FDA for the treatment of wAMD, it is sometimes used off-label. As this analysis focused on FDA-approved anti-VEGF agents, bevacizumab was not considered.

\section{Conclusions}

This analysis suggests that IAI $2 \mathrm{q} 8$ can be cost saving and costeffective compared with Rq4 and ranibizumab PRN, respectively, for the treatment of WAMD in the United States.

\section{Authors}

LUIS HERNANDEZ, MSc; CLIFFORD CELE, BA; and HECTOR TORO-DIAZ, PhD, Modeling and Simulation, Evidera, Waltham, Massachusetts. TEREZA LANITIS, MSc, Modeling and Simulation, Evidera, London, United Kingdom. ANDREA GIBSON, PhD, Medical Affairs Ophthalmology, and ANDREAS KUZNIK, $\mathrm{PhD}$, Health Economics and Outcomes Research, Regeneron Pharmaceuticals, Tarrytown, New York.

AUTHOR CORRESPONDENCE: Luis Hernandez, MSc, Evidera, 500 Totten Pond Rd., Ste. 500, Waltham, MA 02451.

Tel.: 781.960.0249; Email: luis.hernandez@evidera.com.

\section{DISCLOSURES}

This study was funded by Regeneron Pharmaceuticals, the manufacturer of aflibercept. Hernandez, Lanitis, Cele, and Toro-Diaz are employed by Evidera, which received funding from Regeneron Pharmaceuticals to conduct this study. Gibson and Kuznik are employed by and own stock in Regeneron Pharmaceuticals.

\section{REFERENCES}

1. Pascolini D, Mariotti SP, Pokharel GP, et al. 2002 global update of available data on visual impairment: a compilation of population-based prevalence studies. Ophthalmic Epidemiol. 2004;11(2):67-115.

2. Friedman DS, O'Colmain BJ, Munoz B, et al. Prevalence of agerelated macular degeneration in the United States. Arch Ophthalmol. 2004;122(4):564-72

3. National Institutes of Health, National Eye Institute. Age-related macular degeneration (AMD). Available at: https://nei.nih.gov/eyedata/amd. Accessed January 17,2018

4. Jager RD, Mieler WF, Miller JW. Age-related macular degeneration. N Engl J Med. 2008;358(24):2606-17.

5. Heier JS, Brown DM, Chong V, et al. Intravitreal aflibercept (VEGF trap-eye) in wet age-related macular degeneration. Ophthalmology. 2012;119(12):2537-48

6. Arcinue CA, Ma F, Barteselli G, Sharpsten L, Gomez ML, Freeman WR. One-year outcomes of aflibercept in recurrent or persistent neovascular agerelated macular degeneration. Am J Ophthalmol. 2015;159(3):426-36.

7. Ohr M, Kaiser PK. Aflibercept in wet age-related macular degeneration: a perspective review. Ther Adv Chronic Dis. 2012;3(4):153-61.

8. Mousa SA, Mousa SS. Current status of vascular endothelial growth factor inhibition in age-related macular degeneration. BioDrugs. 2010;24(3):183-94.
9. Eylea (aflibercept) injection. Regeneron. Initial U.S. approval, 2011; revised 2017. Available at: https://www.regeneron.com/sites/default/files/ EYLEA_FPI.pdf. Accessed January 17, 2018.

10. Lucentis (ranibizumab injection) for intravitreal injection. Genentech. Initial U.S. approval, 2006; revised 2017. Available at: https://www.gene. com/download/pdf/lucentis_prescribing.pdf. Accessed January 20, 2018.

11. CATT Research Group. Ranibizumab and bevacizumab for neovascular age-related macular degeneration. N Engl J Med. 2011;364(20):1897-908.

12. Kesselheim AS, Avorn J, Sarpatwari A. The high cost of prescription drugs in the United States: origins and prospects for reform. JAMA. 2016;316(8):858-71.

13. Régnier SA, Malcolm W, Haig J, Xue W. Cost-effectiveness of ranibizumab versus aflibercept in the treatment of visual impairment due to diabetic macular edema: a UK healthcare perspective. Clinicoecon Outcomes Res. 2015;7:235-47.

14. Mitchell P, Annemans L, Gallagher M, et al. Cost-effectiveness of ranibizumab in treatment of diabetic macular oedema (DME) causing visual impairment: evidence from the RESTORE trial. $\mathrm{Br} J$ Ophthal. 2012;96(5):688-93

15. Stein JD, Newman-Casey PA, Mrinalini T, Lee PP, Hutton DW. Costeffectiveness of bevacizumab and ranibizumab for newly diagnosed neovascular macular degeneration. Ophthalmology. 2014;121(4):936-45.

16. Cantrill HL. The diabetic retinopathy study and the early treatment diabetic retinopathy study. Int Ophthalmol Clin. 1984;24(4):13-29.

17. Bailey IL, Lovie JE. New design principles for visual acuity letter charts. Am J Optom Physiol Opt. 1976;53(11):740-45.

18. Ferris FL 3rd, Kassoff A, Bresnick GH, Bailey I. New visual acuity charts for clinical research. Am J Ophthalmol. 1982;94(1):91-96.

19. International Council of Ophthalmology. Visual standards. 2002. Available at: http://www.icoph.org/downloads/visualstandardsreport.pdf. Accessed January 17, 2018.

20. Falkenstein IA, Cochran DE, Azen SP, et al. Comparison of visual acuity in macular degeneration patients measured with snellen and early treatment diabetic retinopathy study charts. Ophthalmology. 2008;115(2):319-23.

21. Schmidt-Erfurth U, Kaiser PK, Korobelnik JF, et al. Intravitreal aflibercept injection for neovascular age-related macular degeneration: ninety-sixweek results of the VIEW studies. Ophthalmology. 2014;121(1):193-201.

22. Fisher DE, Jonasson F, Eiriksdottir G, et al. Age-related macular degeneration and mortality in community-dwelling elders: the age, gene/environment susceptibility Reykjavik study. Ophthalmology. 2015;122(2):382-90.

23. Christ SL, Lee DJ, Lam BL, Zheng DD, Arheart KL. Assessment of the effect of visual impairment on mortality through multiple health pathways: structural equation modeling. Invest Ophth Vis Sci. 2008;49(8):3318-23.

24. Wong TY, Chakravarthy U, Klein R, et al. The natural history and prognosis of neovascular age-related macular degeneration: a systematic review of the literature and meta-analysis. Ophthalmology. 2008;115(1):116-26.

25. Bucher HC, Guyatt GH, Griffith LE, Walter SD. The results of direct and indirect treatment comparisons in meta-analysis of randomized controlled trials. J Clin Epidemiol. 1997;50(6):683-91.

26. Marcus DM. Long-term follow-up of intravitreal aflibercept injection (IAI) for neovascular age-related macular degeneration (nAMD) in an openlabel extension of the VIEWl study. Invest Ophth Vis Sci. 2014;55(13):3943.

27. Max Planck Institute for Demographic Research (Germany), University of California, Berkeley (USA), INED (Paris). The Human Mortality Database. 2015 U.S. life tables. Available at: http://www.mortality.org/cgi-bin/hmd/ country.php?cntr=USA\&level=1. Accessed January 17, 2018.

28. Wittenborn JS, Rein D. Cost of vision problems: the economic burden of vision loss and eye disorders in the United States. 2013. Available at: https://www.preventblindness.org/sites/default/files/national/documents/ Economic\%20Burden\%20of\%20Vision\%20Final\%20Report_130611.pdf. Accessed January 17, 2018. 
29. Gencarelli DM. Average wholesale price for prescription drugs: is there a more appropriate pricing mechanism? NHPF Issue brief no. 775. 2002. Available at: http://www.nhpf.org/library/issue-briefs/IB775_AWP_6-7-02. pdf. Accessed January 17, 2018.

30. Gencarelli DM. One pill, many prices: variation in prescription drug prices in selected government programs. NHPF Issue brief no. 807. 2005. Available at: http://www.nhpf.org/library/issue-briefs/IB807_ DrugPricing_08-29-05.pdf. Accessed January 17, 2018.

31. Sanders GD, Neumann PJ, Basu A, et al. Recommendations for conduct, methodological practices, and reporting of cost-effectiveness analyses: Second Panel on Cost-Effectiveness in Health and Medicine. JAMA. 2016;316(10):1093-103.

32. Institute for Clinical and Economic Review. ICER value framework. Discussion of revision options for v2017-2019. 2017. Available at: http://icerreview.org/wp-content/uploads/2016/02/ICER-Value-Assessment-ProposedUpdates-Webinar-021317.pdf. Accessed January 17, 2017.

33. CATT Research Group, Maguire MG, Martin DF, et al. Five-year outcomes with anti-vascular endothelial growth factor treatment of neovascular age-related macular degeneration: the Comparison of Age-Related Macular Degeneration Treatments Trials. Ophthalmology. 2016;123(8):1751-61.

34. Yanagi Y, Fukuda A, Barzey V, Adachi K. Cost-effectiveness of intravitreal aflibercept versus other treatments for wet age-related macular degeneration in Japan. J Med Econ. 2017;20(2):204-12.

35. Murra Anton ZA. Cost-effectiveness analysis of intravitreal aflibercept compared with ranibizumab-prn in patients with wet age-related macular degeneration (Wamd). Value Health. 2015;18(7):A880. Available at: http:// www.valueinhealthjournal.com/article/S1098-3015(15)02143-9/fulltext. Accessed January 17, 2018.

36. Panchmatia HR, Clements KM, Hulbert E, et al. Aflibercept vs. ranibizumab: cost-effectiveness of treatment for wet age-related macular degeneration in Sweden. Acta Ophthalmol. 2016;94(5):441-48.

37. Tempelaar S, Stephens S, Schouten J. Cost-effectiveness of treatments for wet age-related macular degeneration (Wamd) in The Netherlands, the case of intravitreal aflibercept (Ivt-Afl) and ranibizumab. Value Health. 2015;18(7):A420. Available at: http://www.valueinhealthjournal.com/article/ S1098-3015(15)02630-3/fulltext. Accessed January 17, 2018.

38. National Institute for Health and Care Excellence (NICE). Aflibercept solution for injection for treating wet age-related macular degeneration. Technology appraisal guidance 294. July 24, 2013. Available at: https:// www.nice.org.uk/guidance/ta294/resources/aflibercept-solution-for-injection-fortreating-wet-agerelated-macular-degeneration-82600733390533. Accessed January 20, 2018.

39. Vottonen P, Kankaanpaa E. Cost-effectiveness of treating wet age-related macular degeneration at the Kuopio University Hospital in Finland based on a two-eye Markov transition model. Acta Ophthalmol. 2016;94(7):652-56.
40. Elshout M, van der Reis MI, Webers CA, Schouten JS. The cost-utility of aflibercept for the treatment of age-related macular degeneration compared to bevacizumab and ranibizumab and the influence of model parameters. Graefes Arch Clin Exp Ophthalmol. 2014;252(12):1911-20.

41. Claxton L, Hodgson R, Taylor M, Malcolm B, Pulikottil Jacob R. Simulation modelling in ophthalmology: application to cost effectiveness of ranibizumab and aflibercept for the treatment of wet age-related macular degeneration in the United Kingdom. Pharmacoeconomics. 2017;35(2):237-48.

42. Patel JJ, Mendes MAS, Bounthavong M, Christopher ML, Boggie D, Morreale AP. Cost-utility analysis of bevacizumab versus ranibizumab in neovascular age-related macular degeneration using a Markov model. I Eval Clin Pract. 2012;18(2):247-55

43. Czoski-Murray C, Carlton J, Brazier J, Young T, Papo NL, Kang HK. Valuing condition-specific health states using simulation contact lenses. Value Health. 2009;12(5):793-99.

44. Brown MM, Brown GC, Sharma S, Busbee B. Quality of life associated with visual loss: a time tradeoff utility analysis comparison with medical health states. Ophthalmology. 2003;110(6):1076-81.

45. Brown MM, Brown GC, Sharma S, Landy J, Bakal J. Quality of life with visual acuity loss from diabetic retinopathy and age-related macular degeneration. Arch Ophthalmol. 2002;120(4):481-84.

46. Brown GC. Vision and quality-of-life. Trans Am Ophthalmol Soc. 1999;97:473-511.

47. Brown GC, Sharma S, Brown MM, Kistler J. Utility values and age-related macular degeneration. Arch Ophthalmol. 2000;118(1):47-51.

48. Wolf S, Bandello F, Loewenstein A, et al. Baseline characteristics of the fellow eye in patients with neovascular age-related macular degeneration: post hoc analysis of the VIEW studies. Ophthalmologica. 2016;236(2):95-99.

49. Ghosh W, Wickstead R, Claxton L, et al. The cost-effectiveness of ranibizumab treat and extend regimen versus aflibercept in the UK. Adv Ther 2016;33(9):1660-76

50. Rofagha S, Bhisitkul RB, Boyer DS, Sadda SR, Zhang K, Group S-US. Seven-year outcomes in ranibizumab-treated patients in ANCHOR, MARINA, and HORIZON: a multicenter cohort study (SEVEN-UP). Ophthalmology. 2013;120(11):2292-99.

51. Ferreira A, Sagkriotis A, Olson M, Lu J, Makin C, Milnes F. Treatment frequency and dosing interval of ranibizumab and aflibercept for neovascular age-related macular degeneration in routine clinical practice in the USA. PLOS ONE. 2015;10(7):e0133968.

52. Schmid MK, Bachmann LM, Fäs L, Kessels AG, Job OM, Thiel MA. Efficacy and adverse events of aflibercept, ranibizumab and bevacizumab in age-related macular degeneration: a trade-off analysis. $\mathrm{Br} J$ Ophthalmol. 2015;99(2):141-46.

53. Avastin (bevacizumab) injection, for intravenous use. Genentech. Initial U.S. approval: 2004, revised 2017. Available at: https://www.gene.com/ download/pdf/avastin_prescribing.pdf. Accessed January 20, 2018. 